\title{
First records of Zygnematales (Zygnematophyceae, Streptophyta) for the state of Bahia, Brazil
}

\author{
Ivania Batista de Oliveira ${ }^{1,3}$, Carlos Eduardo de Mattos Bicudo ${ }^{2}$ and Carlos Wallace do Nascimento Moura
}

Submitted: 18 March, 2013. Accepted: 25 July, 2013

\begin{abstract}
This study presents the first taxonomic inventory of the order Zygnematales in the Litoral Norte Environmentally Protected Area, in the state of Bahia, Brazil. Fifty samples of planktonic and periphytic material were collected, from lotic and lentic environments, during the summer (January-March) and winter (June-August) of 2009. We identified 18 taxa distributed among five genera (Cylindrocystis, Mougeotia, Netrium, Spirogyra, and Spirotaenia). Of those 18 taxa, eight were new additions to the Brazilian desmid flora: Mougeotia calcarea; Mougeotia elegantula; Spirogyra gracilis; Cylindrocystis crassa var. elliptica; Cylindrocystis brebissonii var. minor; Cylindrocystis brebissonii var. turgida; Netrium digitus var. parvum; and Netrium oblongum var. oblongum. The geographical distributions of ten taxa were extended to include northeastern Brazil.
\end{abstract}

Key words: Brazil, continental microalgae, desmids, Streptophyta, taxonomy

\section{Introduction}

Zygnematales (Zygnematophyceae, Streptophyta) are characterized by having cell walls composed of a single unit, without pores or other ornamentations. The order comprises approximately 1000 species and 20 genera distributed between two families: Zygnemataceae, comprising 13 filamentous genera whose cells are united at their poles to form filaments; and Mesotaeniaceae (known as saccoderm desmids), comprising seven genera, composed of individual unicellular algae that sometimes form amorphous colonies within an abundant mucilaginous matrix (Gerrath 1993; Parra \& Bicudo 1996; Graham et al. 2009).

To our knowledge, there have been no previous studies of the order Zygnematales in the state of Bahia, Brazil. Therefore, the present study sought to undertake the first taxonomic inventory of genera of the order within the state, with the objective of increasing the knowledge of this taxon in Brazil.

\section{Materials and methods}

The Litoral Norte Environmentally Protected Area (EPA) was created by State Decree no. 1.046, on March 17, 1992. The Litoral Norte EPA covers a coastal strip approximately $144 \mathrm{~km}$ long and $10 \mathrm{~km}$ wide (Fig. 1), including five municipalities (Jandaíra, Esplanada, Conde, Entre Rios, and Mata de São João), thus constituting the second-largest EPA in the state of Bahia.

Fifty periphytic and planktonic algae samples were collected, from lotic and lentic environments, in the summer (January-March) and winter (June-August) of 2009. The collections were made following standard methodologies used in taxonomic studies of continental microalgae (Bicudo \& Menezes 2006).

The metric characteristics of the populations were determined using an ocular micrometer. Photomicrographs were obtained using a digital camera coupled to an optical microscope, and species identifications were based on the specialized literature.

Samples were preserved in Transeau's solution, following Bicudo \& Menezes (2006), and incorporated into the liquid collection at the Herbarium of the (Bahia) State University of Feira de Santana (code, HUEFS).

\section{Results and discussion}

Analyses of the 50 samples allowed the identification of 18 taxa among five genera, of which two belonged to the family Zygnemataceae (Mougeotia and Spirogyra) and three belonged to the family Mesotaeniaceae (Cylindrocystis, Netrium, and Spirotaenia).

\footnotetext{
${ }^{1}$ Universidade Estadual de Feira de Santana, Programa de Pós-Graduação em Botânica, Departamento de Ciências Biológicas, Laboratório de Ficologia, Feira de Santana, BA, Brazil

${ }^{2}$ Instituto de Botânica, Seção de Ecologia, São Paulo, SP, Brazil

${ }^{3}$ Author for correspondence: ivboliveira@gmail.com
} 


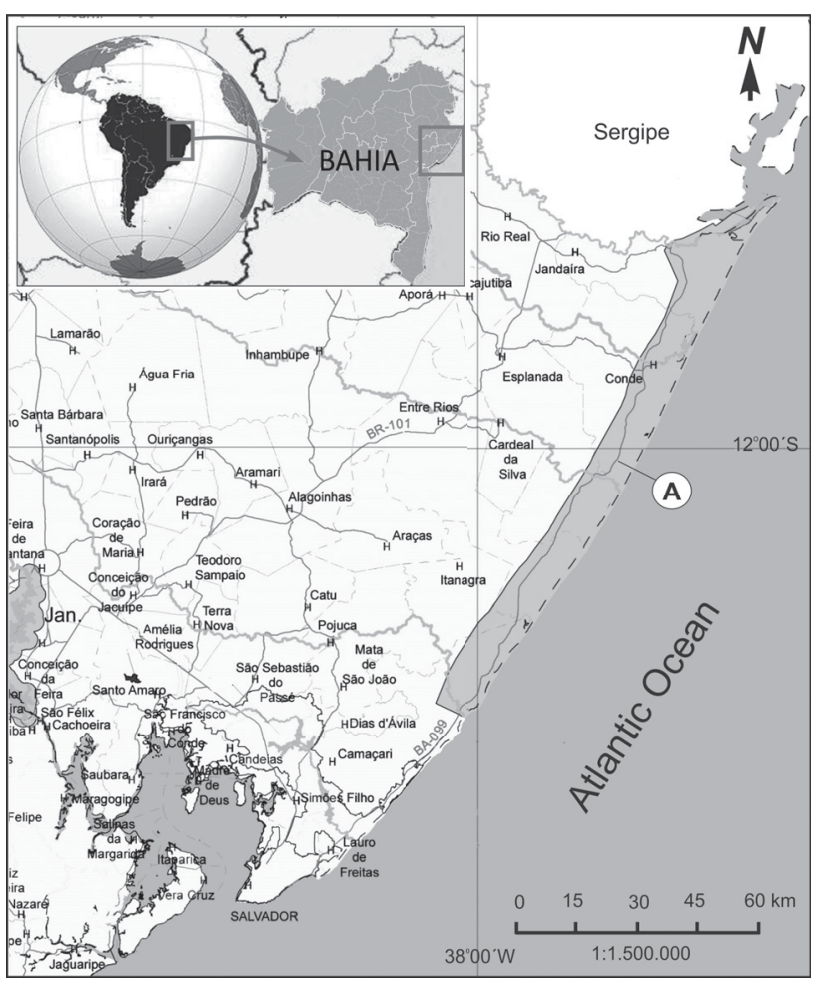

Figure 1. Map of the state of Bahia, Brazil. A. The Litoral Norte Environmentally Protected Area (gray highlighting).

\section{Mougeotia C.Agardh}

Mougeotia calcarea (Cleve) Wittr., Bih. Kgl. Svensk. Vet.Akad. Handl. 1: 40, pl. 2, fig. 1-8. 1872.

Sphaerospermum calcareum Cleve, Nova Acta Reg. Soc. Sci. Upsal.: ser. 3, 6(11): 35, pl. 10, fig. 8-10. 1868.

Fig. 2 and 3.

Simple filaments, vegetative cells 11-12.4 times longer than wide, $145-200 \mu \mathrm{m}$ long, $12-17.5 \mu \mathrm{m}$ wide, lateral margins parallel, straight, smooth, septum region lenticular, angles subquadratic; cell walls hyaline, smooth; chloroplast axial, laminar, pyrenoids 4-5; zygospores 25-30 $\mu \mathrm{m}$ diam., circular outlines, walls smooth, colorless, or light yellow.

Distribution in Brazil: Bahia (present study).

Material selected: Brazil. Bahia: Conde, EPA Litoral Norte, 12/VII/2009, Oliveira et al. s.n. (HUEFS 155730); 02/ VIII/2009, Oliveira \& Moura s.n. (HUEFS 155796).

Morphologically, Mougeotia calcarea is similar to $M$. parvula Hassall, although it differs by having smaller cell dimensions and dark brown zygospores (Transeau 1926, 1951; Zarina et al.2008).

Mougeotia elegantula Wittr., Bih. Kgl. Svensk. Vet.-Akad. Handl. 1: 40, pl. 3, fig. 5-8. 1872.

Fig. 4 and 5.

Simple filaments, vegetative cells 12-25 times longer than wide, 50-150 $\mu \mathrm{m}$ long, 4-6 $\mu \mathrm{m}$ wide, lateral margins parallel, straight, smooth, septum region straight; cell walls hyaline, smooth; chloroplast axial, laminar, twisted; pyrenoids 4-8 in median series; zygospores $26-20 \mu \mathrm{m}$ long, $22-25 \mu \mathrm{m}$ wide, quadrangular in outline, margins concave, walls smooth, hyaline.

Distribution in Brazil: Bahia (present study).

Material selected: Brazil. Bahia: Conde, EPA Litoral Norte, 12/VII/2009, Oliveira et al. s.n. (HUEFS 155723); 02/ VIII/2009, Oliveira \& Moura s.n. (HUEFS 155796, HUEFS 155799, HUEFS 155815).

Mougeotia elegantula demonstrates conjugation of the scalariform type, with quadratic zygospores, cruciate with rounded angles. In terms of its morphology, M. elegantula can be compared to M. virescens (Hassall) Borge, although differing from the latter by having larger zygospores with deeply concave sides, and larger vegetative cells.

Mougeotia cf. gotlandica (Cleve) Wittr., Bih. Kgl. Svensk. Vet.-Akad. Handl. 1: 39. 1872.

Mesocarpus gotlandicus Cleve, Monogr. öf. Zygn. 31. 1868. Fig. 6.

Simple filaments, vegetative cells 4-8 times longer than wide, 67.5-165 $\mu \mathrm{m}$ long, 16-20 $\mu \mathrm{m}$ wide, lateral margins parallel, straight, smooth; septum region straight; cell walls hyaline, smooth; neither chloroplasts nor pyrenoids were observed; zygospore 30-35 $\mu \mathrm{m}$ diam., outline circular-ovate, wall smooth, hyaline.

Distribution in Brazil: São Paulo (Pereira \& Branco 2010), Bahia (present study).

Material selected: Brazil. Bahia: Conde, EPA Litoral Norte, 11/I/2009, Oliveira \& Moura s.n. (HUEFS 155610); 14/III/2009, Oliveira \& Moura s.n. (HUEFS 155710); 14/ III/2009, Oliveira \& Farias s.n. (HUEFS 155787); $12 /$ VII/2009, Oliveira et al. s.n. (HUEFS 155723).

Mougeotia cf. gotlandica shows scalariform type conjugation, with globose zygospores. We only tentatively identified the present material due to the absence of chloroplasts in the cells and only small numbers of specimens in the samples.

\section{Spirogyra Link}

Spirogyra gracilis (Hassall) Kütz., Sp. Alg. 438. 1849.

Zygnema gracile Hassall, Brit. Freshw. Algae 1: 148, pl. 30, fig. 5-6. 1845.

Fig. 7 and 8.

Simple filaments, vegetative cells 2.8-4 times longer than wide, $75-100 \mu \mathrm{m}$ long, $22-25 \mu \mathrm{m}$ wide, lateral margins parallel, straight, smooth, septum region lenticular, angles subquadratic; cell walls hyaline, smooth; chloroplasts 2 , ribbon-like, parietal, helicoidal, with 3-4 twists, various pyrenoids in each chloroplast; zygospores 55-58 $\mu \mathrm{m}$ long, 32.5-35 $\mu \mathrm{m}$ wide; outline ellipsoidal, cell walls smooth, brownish.

Distribution in Brazil: Bahia (present study).

Material selected: Brazil. Bahia: Conde, EPA Litoral Norte, 11/I/2009, Oliveira \& Moura s.n. (HUEFS 155603); 15/II/2009, Oliveira \& Moura s.n. (HUEFS 155637); 01/ 
III/2009, Oliveira \& Farias s.n. (HUEFS 155665); 11/ VII/2009, Oliveira et al. s.n. (HUEFS 155714), 02/VIII/2009, Oliveira \& Moura s.n. (HUEFS 155809).

Morphologically, Spirogyra gracilis can be compared to S. acantophora (Skuja) Czurda but differs from it by having larger vegetative and reproductive cells and zygospores with irregular and spiny walls. During the analyses of S. gracilis, we observed some specimens in the process of scalariform conjugation, with swollen gametangia and ellipsoidal zygospores with rounded poles.
Cylindrocystis Meneghini ex Ralfs

Cylindrocystis brebissonii Menegh. ex De Bary var. brebissonii, Unter. Conjugaten. 35, pl. 7, fig. E1-22. 1858.

Fig. 9 and 10.

Cells 3.5-4 times longer than wide, 70-90 $\mu \mathrm{m}$ long, 20-22.5 $\mu \mathrm{m}$ wide, lateral margins parallel, poles truncate-rounded; cell walls smooth; 2 axial chloroplasts, star-shaped in transversal section, lamellate, 1 pyrenoid per chloroplast. Distribution in Brazil: Amazonas (Förster 1969; Scott et al. 1965); Bahia (present study); Minas Gerais (Wille 1884);
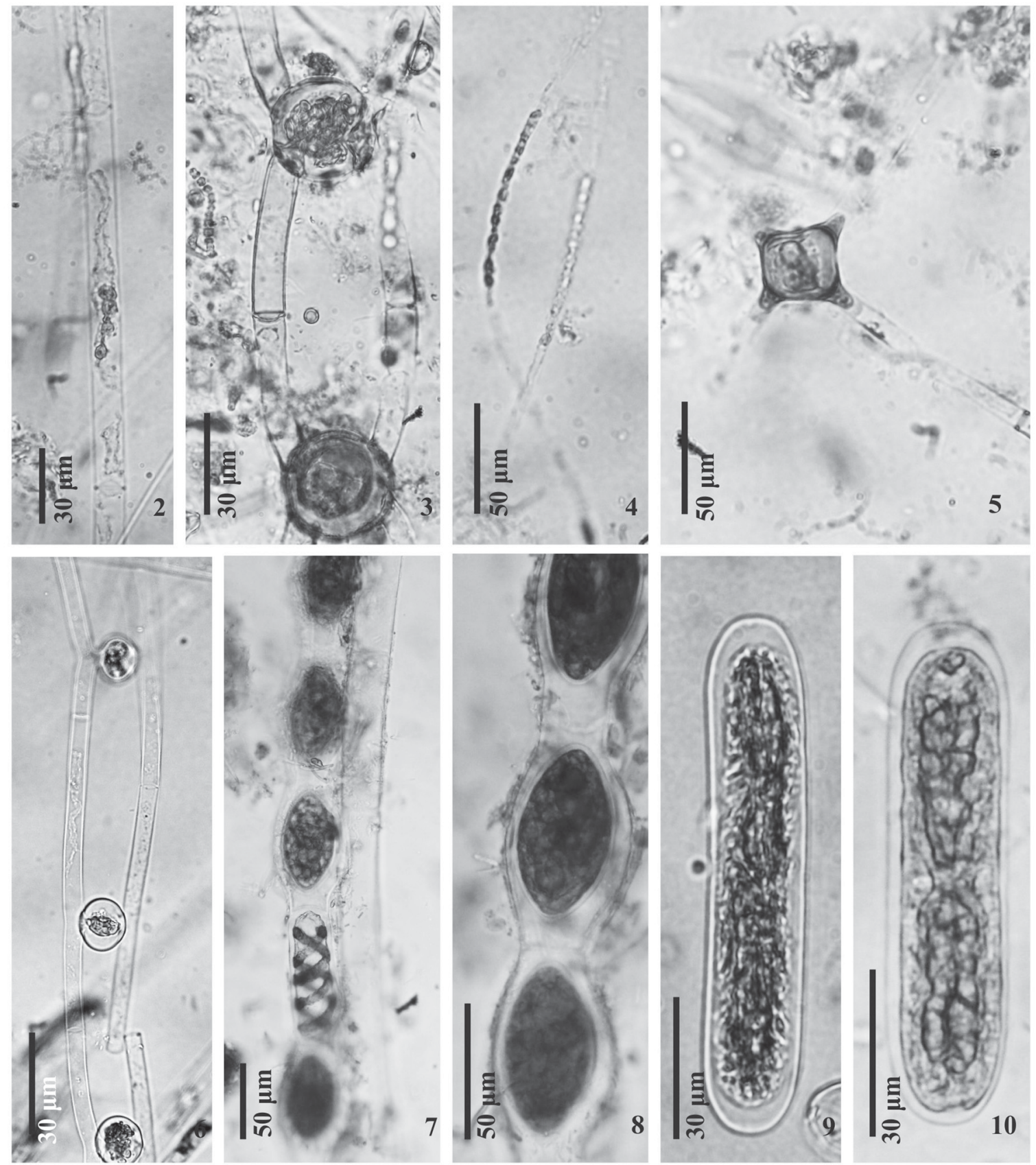

Figures 2-10. 2. Mougeotia cf. calcarea (Cleve) Wittr.; 3. Zygospore; 4. Mougeotia elegantula Wittr.; 5. M. elegantula zygospore; 6. Mougeotia cf. gotlandica (Cleve) Wittr.; 7,8. Spirogyra gracilis (Hassall) Kütz.; 9,10. Cylindrocystis brebissonii Menegh. ex De Bary var. brebissonii. 
Paraná (Felisberto \& Rodrigues 2008); Rio de Janeiro (Bicudo \& Bicudo 1969); Rio Grande do Sul (Rosa et al. 1987); São Paulo (Borge 1918; Wittrock \& Nordstedt 1882; Bicudo 1969).

Material selected: Brazil. Bahia: Conde, EPA Litoral Norte, 28/II/2009, Oliveira \& Farias s.n. (HUEFS 155642); 12/VII/2009, Oliveira et al. s.n. (HUEFS 155701, HUEFS 155726), 26/VII/2009, Oliveira \& Farias s.n. (HUEFS 155774).

Felisberto \& Rodrigues (2008) reported smaller cell dimensions $(47.3-71 \times 14-14.4 \mu \mathrm{m})$ for samples from the state of Paraná as compared with those from Bahia.

Cylindrocystis brebissonii Menegh. ex De Bary var. minor West \& G.S. West, Trans. Roy. Irish. Acad.: ser. B, 32(1): 20, pl. 2, fig. 7. 1902.

Fig. 11.

Cells 1.7-2 times longer than wide, 30-35 $\mu \mathrm{m}$ long, $16-$ $17.5 \mu \mathrm{m}$ wide, lateral margins parallel, poles truncate-rounded; cell walls smooth; 2 axial chloroplasts, star-shaped in transversal section; 1 pyrenoid per chloroplast.

Distribution in Brazil: Bahia (present study).

Material selected: Brazil. Bahia: Conde, EPA Litoral Norte, 26/VII/2009, Oliveira \& Farias s.n. (HUEFS 155774, HUEFS 155782); 02/VIII/2009, Oliveira \& Moura s.n. (HUEFS 155796, HUEFS 155799).

Cylindrocystis brebissonii var. minor differs from the typical variety of this species only by having smaller cell dimensions. Morphologically, the variety minor may be confused with Actinotaenium truncatum (Bréb. ex Ralfs) Teiling ex Růžička \& Pouzar, but the latter differs by having more rounded apical margins and a slight median constriction.

Cylindrocystis brebissonii (Menegh. ex Ralfs) De Bary var. turgida Schmidle, Österreich Botanische Zeitschrift 45: 309, pl. 14, fig. 15. 1895.

Fig. 12.

Cells 1.5-1.8 times longer than wide, 40-44 $\mu \mathrm{m}$ long, 22-29 $\mu \mathrm{m}$ wide, poles rounded; lateral margins straight, subparallel to each other; cell walls smooth; 2 axial chloroplasts, star-shaped in transversal section, 1 pyrenoid per chloroplast.

Distribution in Brazil: Bahia (present study).

Material selected: Brasil. Bahia: Conde, EPA Litoral Norte, 01/III/2009, Oliveira \& Farias s.n. (HUEFS 155676); 14/III/2009, Oliveira \& Moura s.n. (HUEFS 155705); 12/ VII/2009, Oliveira et al. s.n. (HUEFS 155676), 26/VII/2009, Oliveira \& Farias s.n. (HUEFS 155745).

Cylindrocystis brebissonii var. turgida differs from the type-variety of the species by having a generally cylindrical shape, being 1.5-1.8 times longer than wide.

Cylindrocystis crassa De Bary var. crassa, Unter. Conjugaten. 37, pl. 7C, fig. 1-12. 1858.

Fig. 13.
Cells 1.4-1.5 times longer than wide, 30-32.5 $\mu \mathrm{m}$ long, 20-22.5 $\mu \mathrm{m}$ wide, poles rounded; lateral margins straight, parallel to each other, cell walls smooth; 2 axial chloroplasts, star-shaped in transversal section, 1 pyrenoid per chloroplast.

Distribution in Brazil: Amazonas (Förster 1969); Bahia (present study); Pará (Förster 1969).

Material selected: Brazil. Bahia: Conde, EPA Litoral Norte, 15/II/2009, Oliveira \& Moura s.n. (HUEFS 155632); 14/III/2009, Oliveira \& Moura s.n. (HUEFS 155710); 26/ VII/2009, Oliveira et al. s.n. (HUEFS 155726), 02/VIII/2009, Oliveira \& Moura s.n. (HUEFS 155797).

During the current study, specimens of Cylindrocystis crassa var. crassa always occurred isolated and never within a mucilaginous sheath as often described in the literature. Förster (1969) reported specimens from the Amazon with smaller dimensions $(23 \times 18 \mu \mathrm{m})$ than those encountered in the present study.

Cylindrocystis crassa De Bary var. elliptica West \& G.S. West, Trans. Linn. Soc. London: ser. 2, 5: 48, pl. 5, fig. 27. 1895.

Fig. 14-16.

Cells 1.2-1.3 times longer than wide, 25-27.5 $\mu \mathrm{m}$ long, 18.5-22.5 $\mu \mathrm{m}$ wide, margins smooth, poles widely rounded; cell walls smooth; 2 axial chloroplasts, star-shaped in transversal section, with prominent longitudinal projections, 1 pyrenoid, central.

Distribution in Brazil: Bahia (present study).

Material selected: Brazil. Bahia: Conde, EPA Litoral Norte, 14/III/2009, Oliveira \& Moura s.n. (HUEFS 155689, HUEFS 155710); 26/VII/2009, Oliveira \& Farias s.n. (HUEFS 155769); 12/VII/2009, Oliveira et al. (HUEFS 155726); 02/ VIII/2009, Oliveira \& Moura s.n. (HUEFS 155821).

Cylindrocystis crassa var. elliptica differs from the typical variety of this species by having subcircular cells with widely rounded poles. In terms of its morphology, this variety can be confused with Mesotaenium chlamydosporum De Bary var. violascens (De Bary) Willi Krieg., although the latter has smooth and straight parietal laminar chloroplasts, and its cell walls are violet colored.

Netrium (Nägeli) Itzigsohn \& Rothe emend. Lütkem. Netrium digitus (Ralfs) Itzigsohn \& Rothe var. digitus, In Rabenhorst, Alg. Sachsen no 508. 1856.

Penium digitus Ralfs, Brit. Desm.: 150, pl. 25, fig. 3. 1848. Fig. 17.

Cells straight, fusiform-truncated, 4-5.8 times longer than wide, 150-320 $\mu \mathrm{m}$ long, 37.5-55 $\mu \mathrm{m}$ wide, gradually narrowing towards the apex, poles truncate-rounded, lateral margins equally convex; cell walls smooth; 2 axial chloroplasts, with prominent and denticulate longitudinal projections, pyrenoids 2-3 in median series per chloroplast. Distribution in Brazil: Amazonas (Scott et al. 1965); Bahia (present study); Mato Grosso (De-Lamonica-Freire \& 
Heckman 1996); Paraná (Felisberto \& Rodrigues 2008); Rio de Janeiro (Bicudo \& Bicudo 1969; Sophia 1991); Rio Grande do Sul (Ungaretti 1981; Rosa et al. 1987, 1988; Franceschini 1992).

Material selected: Brasil. Bahia: Conde, EPA Litoral Norte, 11/I/2009, Oliveira \& Moura s.n. (HUEFS 155602); 14/II/2009, Oliveira \& Moura s.n. (HUEFS 155614); 14/ III/2009, Oliveira \& Farias s.n. (HUEFS 155684); 11/ VII/2009, Oliveira et al. s.n. (HUEFS 155715), 02/VIII/2009, Oliveira \& Moura s.n. (HUEFS 155797).

Netrium digitus var. digitus can be identified relatively easily due to a number of unique characteristics, such as the fusiform-truncated shape of its cells; smooth, hyaline cell walls; and chloroplasts with denticulate projections. Felisberto \& Rodrigues (2008) reported smaller cell dimensions

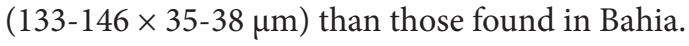

Netrium digitus (Ehrenb. ex Ralfs) Itzigsohn \& Rothe var. lamellosum (Bréb.) Grönblad, Acta Soc. Fauna Flora Fenn. 47(4): 13. 1920.

Penium lamellosum Bréb., Mém. Soc. Sci. Nat. Cherbourg 4: 146, pl. 2, fig. 34. 1856.

Fig. 18.

Cells straight, elliptical-elongated, 4.5-5.5 times longer than wide, 202.5-280 $\mu \mathrm{m}$ long, 44-50 $\mu \mathrm{m}$ wide, slight median constriction, lateral margins subparallel to each other, poles rounded; cell walls smooth; 2 axial chloroplasts, with prominent lateral projections, pyrenoids 2-3 in median series per chloroplast.

Distribution in Brazil: Bahia (present study); Pará (Grönblad 1945; Scott et al. 1965); Rio Grande do Sul (Borge 1903); São Paulo (Bicudo 1969).

Material selected: Brazil. Bahia: Conde, EPA Litoral Norte, 14/II/2009, Oliveira \& Moura s.n. (HUEFS 155623); 15/II/2009, Oliveira \& Moura s.n. (HUEFS 155637), 14/ III/2009, Oliveira \& Moura s.n. (HUEFS 155680); 12/ VII/2009, Oliveira et al. s.n. (HUEFS 155722, HUEFS 155726), 26/VII/2009, Oliveira \& Farias s.n. (HUEFS 155748).

Netrium digitus var. lamellosum differs from the typical variety of the species by having chloroplasts with lamellae with prominent lateral projections, and a slight depression on each of the lateral margins of the cell.

Bourrelly \& Coute (1991) reported the existence of specimens with significantly larger cell dimensions $(520 \times$ $90 \mu \mathrm{m}$ ), almost twice the size of those collected in Bahia.

Netrium digitus (Ehrenb. ex Ralfs) Itzigsohn \& Rothe var. naegelii (Bréb. ex W.Archer) Willi Krieg. In Rabenhorst, Krypt.-Fl. Deutschland, Alg. 13(1):218, pl. 8, fig. 4-5. 1937. Penium naegelii Bréb. ex W. Archer in Pritchard, Infusoria. 751. 1861.

Fig. 19.

Cells elongated, fusiform, 5-6.5 times longer than wide, $220-250 \mu \mathrm{m}$ long, 40-42.5 $\mu \mathrm{m}$ wide, lateral margins slightly convex, narrowing toward the apex, poles truncate-rounded; cell walls hyaline, smooth; 2 axial chloroplasts with prominent projections; pyrenoids 2-3 in median series per chloroplast.

Distribution in Brazil: Amazonas (Scott et al. 1965); Bahia (present study); Mato Grosso (Borge 1903); Pará (Grönblad 1945); São Paulo (Børgesen 1890; Borge 1918; Bicudo 1969). Material selected: Brazil. Bahia: Conde, EPA Litoral Norte, 11/I/2009, Oliveira \& Moura s.n. (HUEFS 155602); 14/II/2009, Oliveira \& Moura s.n. (HUEFS 155612);26/VII/2009, Oliveira \& Farias s.n. (HUEFS 155787, HUEFS 155745).

Netrium digitus var. naegelii differs from the typical variety of the species by having smaller and relatively narrow cells, although it is sometimes difficult to establish limits between the two varieties given the existence of intermediate forms. Bicudo (1969) reported smaller cell dimensions (85$120 \times 23.5-33.5 \mu \mathrm{m})$ for material collected in the state of São Paulo as compared with the material collected in Bahia.

Netrium digitus (Ehrenb. ex Ralfs) Itzigsohn \& Rothe var. parvum Borge, Ark. Bot. 19(17): 14, pl. 1, fig. 19. 1925.

Fig. 20.

Cells fusiform, 2.5-2.6 times longer than wide, 100-110 $\mu \mathrm{m}$ long, 39-42.5 $\mu \mathrm{m}$ wide, lateral margins more or less parallel to each other, poles truncate-rounded; cell walls smooth; 2 axial chloroplasts, with prominent lateral projections, 2-3 pyrenoids in median series per chloroplast. Distribution in Brazil: Bahia (present study).

Material selected: Brazil. Bahia: Conde, EPA Litoral Norte, 11/I/2009, Oliveira \& Moura s.n. (HUEFS 155603); 14/II/2009, Oliveira \& Moura s.n. (HUEFS 155618); 01/ III/2009, Oliveira \& Farias s.n. (HUEFS 155674); 02/ VIII/2009, Oliveira \& Moura s.n. (HUEFS 155826).

The differences between the parvum variety and the typical variety of this species are its truncate-rounded poles, chloroplasts with prominent lateral projections, and relatively smaller cell dimensions.

The var. parvum could be initially confused with Closterium closterioides (Ralfs) Louis \& Peeters var. intermedium (Roy \& Bisset) Růžička, although the latter has lamellate chloroplasts and apical vacuole containing one or more granules of calcium sulfate.

Netrium interruptum (Bréb. ex Ralfs) Lütkem. var. interruptum, Beitr. Biol. Pflanzen 8: 407. 1902.

Penium interruptum Bréb. ex Ralfs, Brit. Desmidieae. 151, pl. 25, fig. 4. 1848.

Fig. 21.

Cells cylindrical, 5-5.4 times longer than wide, 125-135 $\mu \mathrm{m}$ long, $25 \mu \mathrm{m}$ wide, apex $12.5 \mu \mathrm{m}$ wide, lateral margins straight, parallel to each other, narrowing towards the apex, poles rounded; cell walls smooth; 2 axial chloroplasts per semicell, lamellate; pyrenoids 4 in each chloroplast.

Distribution in Brazil: Amazonas (Scott et al. 1965); Bahia (present study). 

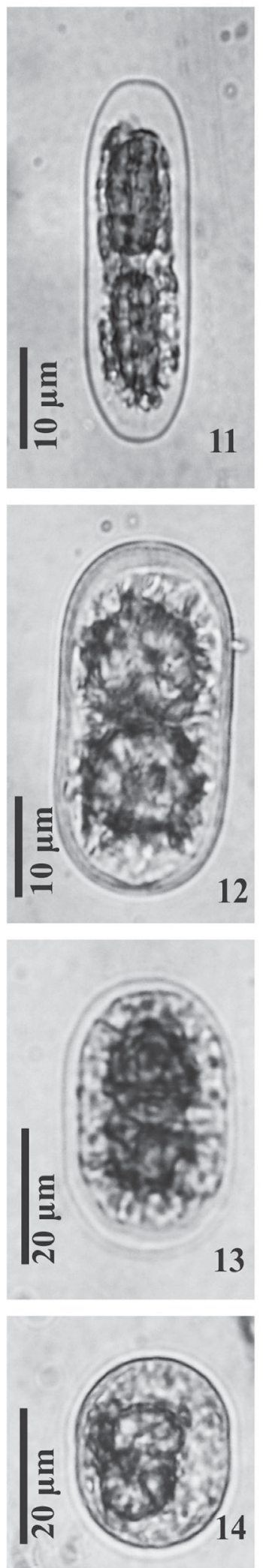
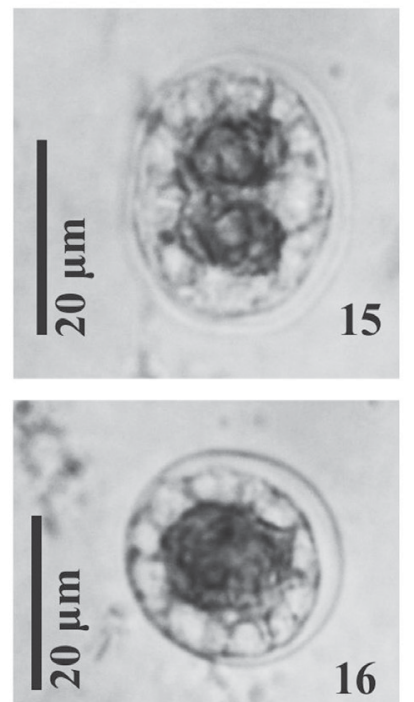

16

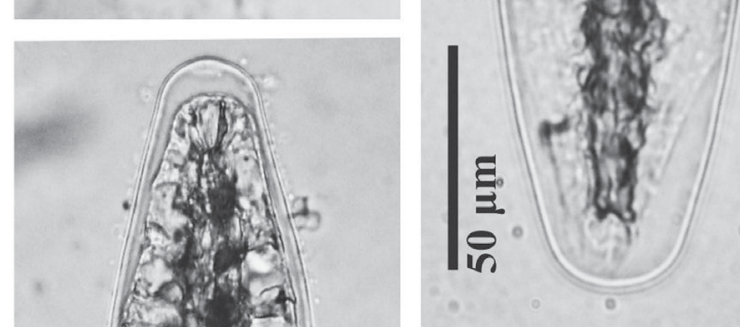

17
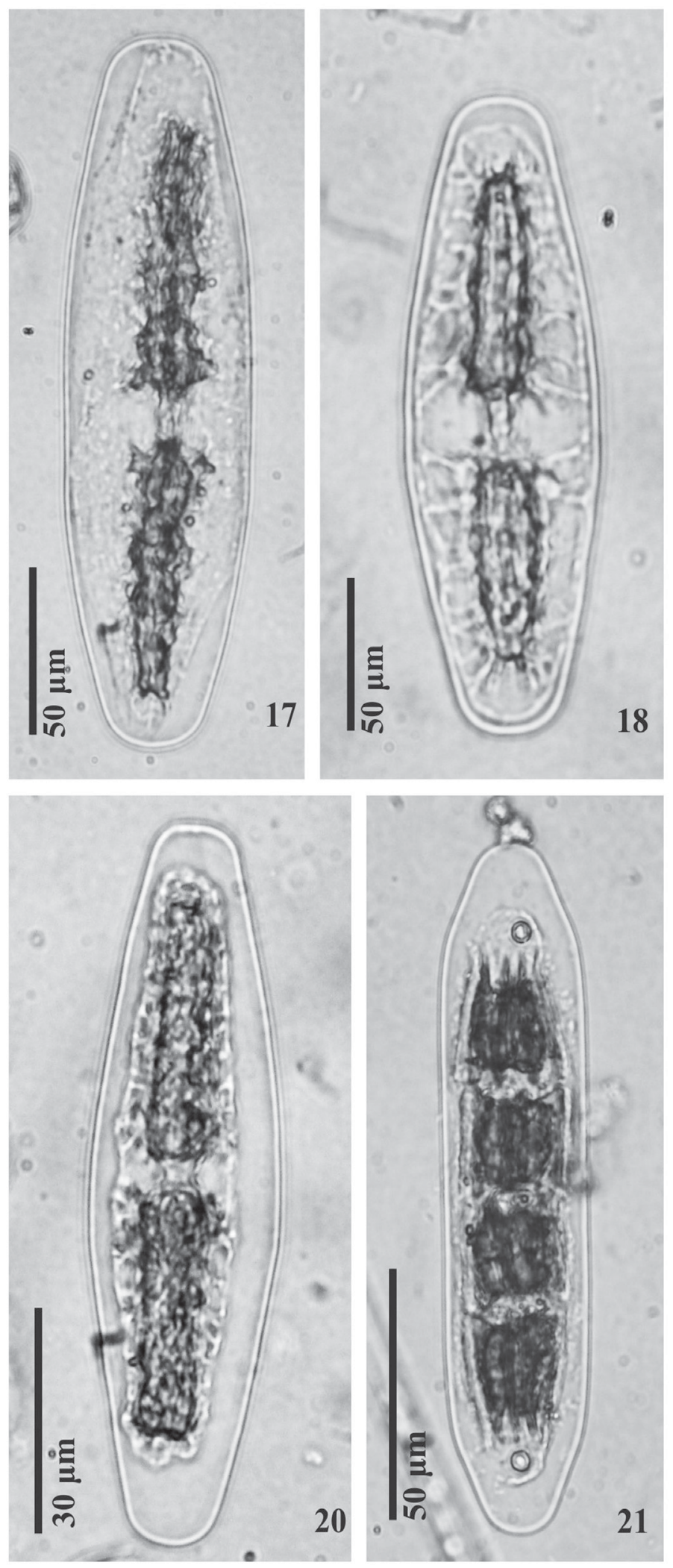

Figures 11-21. 11. Cylindrocystis brebissonii Menegh. ex De Bary var. minor West \& G.S.West; 12. Cylindrocystis brebissonii (Menegh. ex Ralfs) De Bary var. turgida Schmidle; Figure 13. Cylindrocystis crassa De Bary var. crassa; 14,15. Cylindrocystis crassa De Bary var. eliptica West \& G.S.West; 16. C. crassa var. eliptica, apical view, detail; 17. Netrium digitus (Ralfs) Itzigsohn \& Rothe var. digitus; 18. Netrium digitus (Ehrenb. ex Ralfs) Itzigsohn \& Rothe var. lamellosum (Bréb.) Grönblad; 19. Netrium digitus (Ehrenb. ex Ralfs) Itzigsohn \& Rothe var. naegelii (Bréb. ex W.Archer) Willi; 20. Netrium digitus (Ehrenb. ex Ralfs) Itzigsohn \& Rothe var. parvum (Borge) Willi Krieg.; 21. Netrium interruptum (Bréb. ex Ralfs) Lütkem. var. interruptum. 
Material selected: Brazil. Bahia: Conde, EPA Litoral Norte, 14/II/2009, Oliveira \& Moura s.n. (HUEFS 155618); 15/II/2009, Oliveira \& Moura s.n. (HUEFS 155638); 14/ III/2009, Oliveira \& Moura s.n. (HUEFS 155701); 26/ VII/2009, Oliveira et al. s.n. (HUEFS 155726).

Scott et al. (1965) and Croasdale \& Flint (1986) identified specimens with smaller cell dimensions $(330 \times 34 \mu \mathrm{m}$ and $150-400 \times 30-82 \mu \mathrm{m}$, respectively) than the material collected in Bahia.

Netrium interruptum (Ralfs) Lütkem. var. minor (Borge) Willi Krieg. In Rabenhorst, Krypt.-Fl. Deutschland, Alg. 13(1): 223, pl. 8, fig. 10. 1937.

Penium interruptum Bréb. f. minor Borge, Ark. Bot. 8(13): 2, pl. 1, fig. 10. 1909.

Fig. 22.

Cells cylindrical, 3.4-4.4 times longer than wide, 85-110 $\mu \mathrm{m}$ long, ca. $25 \mu \mathrm{m}$ wide, apex ca. $12.5 \mu \mathrm{m}$ wide, lateral margins straight, parallel to each other, narrowing slightly towards the apex, poles truncated; cell walls smooth; 2 axial chloroplasts in each semicell, lamellate, pyrenoids 4 in each chloroplast.

Distribution in Brazil: Bahia (present study); Pará (Förster 1969); Rio de Janeiro (Bicudo \& Picelli-Vicentim 1988); São Paulo (Bicudo 1969).

Material selected: Brazil. Bahia: Conde, EPA Litoral Norte, 11/I/2009, Oliveira \& Moura s.n. (HUEFS 155610); 14/III/2009, Oliveira \& Moura s.n. (HUEFS 155712); 12/ VII/2009, Oliveira et al. s.n. (HUEFS 155723), 02/VIII/2009, Oliveira \& Moura s.n. (HUEFS 155805).

Netrium interruptum var. minor differs from the typical variety of the species only by having smaller cell dimensions.

Netrium oblongum (De Bary) Lütkem. var. oblongum, Cohn's Beitr. Biol. Pflanzen 8: 407. 1902.

Penium oblongum De Bary, Unter. Fam. Conjug. 73, pl. 7G, fig. 1, 3. 1858.

Fig. 23.

Cells oblong, 4.1-4.4 times longer than wide, 79-85 $\mu \mathrm{m}$ long, $18-20 \mu \mathrm{m}$ wide, lateral margins straight, parallel to each other, narrowing toward the apex, poles truncate-rounded; cell walls smooth; 2 axial chloroplasts, with lamellae. Distribution in Brazil: Bahia (present study).

Material selecionado: Brasil. Bahia: Conde, EPA Litoral Norte, 28/III/2009, Oliveira \& Farias s.n. (HUEFS 155642); 14/III/2009, Oliveira \& Moura s.n. (HUEFS 155685); 12/ VII/2009, Oliveira et al. s.n. (HUEFS 155732).

Netrium oblongum var. oblongum can be identified relatively easily because of unique characteristics, such as: (1) the oblong shape of its semicells; (2) chloroplasts with lamellae saw-edged along their entire length.

Netrium oblongum (De Bary) Lütkem. var. cylindricum West \& G.S. West, J. Bot. 41: 40, pl. 446, fig. 10. 1903.

Fig. 24.
Cells cylindrical, 2.8-3 times longer than wide, 50-56 $\mu \mathrm{m}$ long, $17-19 \mu \mathrm{m}$ wide, lateral margins straight, parallel to each other, narrowing slightly towards the apex, poles truncate-rounded; cell walls hyaline, smooth; 2 axial chloroplasts, with lamellae; pyrenoids 4 in each chloroplast. Distribution in Brazil: Amazonas (Förster 1969); Bahia (present study); São Paulo (Borge 1918).

Material selected: Brazil. Bahia: Conde, EPA Litoral Norte, 15/II/2009, Oliveira \& Moura s.n. (HUEFS 155634); 11/ VII/2009, Oliveira et al. s.n. (HUEFS 155732), 02/VIII/2009, Oliveira \& Moura s.n. (HUEFS 155826).

Netrium oblongum var. cylindricum can be distinguished from the typical variety of the species by having more cylindrical cells, proportionately smaller cell dimensions, and poles that are more rounded.

Spirotaenia Bréb. in Ralfs

Spirotaenia condensata Bréb. ex Ralfs, Brit. Desm. 179, pl. 34, fig. 1. 1848.

Fig. 25.

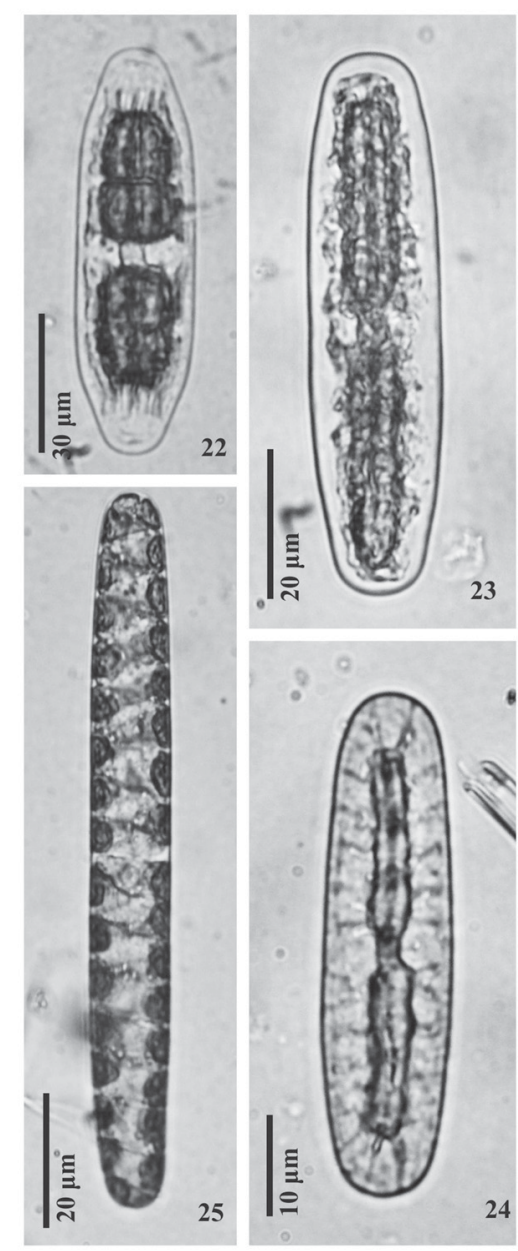

Figures 22-25. 22. Netrium interruptum (Ralfs) Lutkem. var. minor (Borge) Willi Krieg.; 23. Netrium oblongum (de Bary) Lütkem. var. oblongum; 24. Netrium oblongum (de Bary) Lütkem. var. cylindricum West \& G.S. West; 25. Spirotaenia condensata Bréb. ex Ralfs. 
Cells cylindrical, 8-9.5 times longer than wide, $120-190 \mu \mathrm{m}$

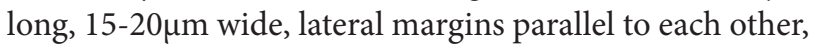
smooth, poles rounded; cell walls hyaline, smooth; chloroplast ribbon-shaped, parietal, twisted 15 times, pyrenoids 2-3 in median series.

Distribution in Brazil: Amazonas (Förster 1969); Bahia (present study); Minas Gerais (Soares et al. 2007); Rio de Janeiro (Bicudo \& Bicudo 1969).

Material selected: Brazil. Bahia: Conde, EPA Litoral Norte, 11/I/2009, Oliveira \& Moura s.n. (HUEFS 155603, HUEFS 155610); 25/VII/2009, Oliveira \& Farias s.n. (HUEFS 155741); 02/VIII/2009, Oliveira \& Moura s.n. (HUEFS 155809).

Spirotaenia condensata can be identified relatively easily due to a number of unique characteristics: cells almost perfectly cylindrical; chloroplasts ribbon-shaped and wide, helicoidal; and poles rounded.

\section{Acknowledgments}

The authors would like to thank the Botanical Institute of the São Paulo State Department of the Environment, for the logistical support provided. This study received financial support from the Coordenação de Aperfeiçoamento de Pessoal do Ensino Superior (CAPES, Office for the Advancement of Higher Education; doctoral grant to IBO), as well as from the Universidade Estadual de Feira de Santana Graduate Program in Botany, which provided the financial resources needed in order to make the field collections.

\section{References}

Bicudo, C.E.M. 1969. Contribution to the knowledge of the state of São Paulo, Brazil (including from the state of Minas Gerais). Nova Hedwigia 17(1-4): 443-549.

Bicudo, C.E.M. \& Bicudo, R.M.T. 1969. Algas da lagoa das Prateleiras, Parque Nacional do Itatiaia, Brasil. Rickia 4: 1-40.

Bicudo, C.E.M. \& Picelli-Vicentim, M.M. 1988. Ficoflórula do campo de esfagno das prateleiras, Parque Nacional de Itatiaia, sul do Brasil. Revista Brasileira de Biologia 48: 15-28.

Bicudo, C.E.M. \& Menezes, M. 2006. Gêneros de algas de águas continentais do Brasil: chave para identificação e descrições. 2 ed. RiMa, São Carlos.

Borge, O. 1903. Die Algen der ersten Regnellschen Expedition, 2: Desmidiaceae. Arkiv för Botanik 1: 71-138.

Borge, O. 1918. Die von Dr.A. Löfgren in São Paulo gessammelten Süsswasseralgen. Arkiv för Botanik 15(13): 1-108.

Børgesen, O. 1890. Desmidiaceae. Pp. 2-5. In: Warming, E. (Ed.). Symbolae ad floram Brasiliae centralis cognoscendam. Videnskabelige Meddelelser fra den naturhistoriske Forening i Kjöbenhavn 46: 930-958.
Bourrelly, P. \& Couté, A. 1991. Desmidiées de Madagascar (Chlorophyta, Zygophyceae). Bibliotheca Phycologica 86: 1-348.

De-Lamonica-Freire, E.M. \& Heckman, C.W. 1996. The seasonal succession of biotic communities in wetlands of the tropical wet-and-dray climatic zone: III. The algal communities in the Pantanal of Mato Grosso, Brazil, with a comprehensive list of the known species and revision of two desmid taxa. Institut für Hydrobiologie und Fischereiwissenschaft 81(2): 253-280.

Felisberto, S.A. \& Rodrigues, L. 2008. Desmidiaceae, Gonatozygaceae e Mesotaeniaceae na comunidade perifítica do reservatório de Salto do Vau (Bacia do Rio Iguaçu, PR). Hoehnea 35: 235-254.

Förster, K. 1969. Amazonische desmidien, 1. Amazoniana 2(1-2): 5-116.

Franceschini, L.M. 1992. Algues d'eau douce de Porto Alegre, Brésil (les Diatomophycées exclues). Bibliotheca Phycologica. B. 92: 1-81.

Gerrath, J.F. 1993. The biology of desmids: a decade of progress. Pp. 77192. In: Round, F. \& Chapman, D.J. (Eds.). Progress in Phycological Research 9. Biopress Ltd., Bristol.

Graham, L.; Graham, J. \& Wilcox, L. 2009. Algae, 2e. Benjamin Cummings (Pearson), San Francisco, CA.

Grönblad, R. 1945. De algus brasiliensibus: praecipue Desmidiacius, in regione inferiore fluminis Amazonas, Acta Societatis Scientiarum Fennicae, nov., ser. B. Helsinforsiave, 2(6): 1- 43.

Parra, O. \& Bicudo, C.E.M. 1996. Algas de aguas continentales: Introducción a la Biología y sistemática. Ediciones Universidad de Concepción.

Pereira, J.L. \& Branco, L.H.Z. 2010. Macroalgas em nascentes e arredores de riachos na região noroeste do Estado de São Paulo. Hoehnea 37(3): 435-444.

Rosa, Z.M.; Torgan, L.C.; Lobo, E.A. \& Herzog, L.A.W. 1987. Ficoflórula de ambientes lênticos, estudo preliminar da Região de Charqueadas, Rio Grande do Sul, brasil com vistas à avaliação ambiental. Acta Botânica Brasilica 1(2) 165-188.

Rosa, Z.M.; Torgan, L.C.; Lobo, E.A. \& Herzog, L.A.W. 1988. Análise da estrutura de comunidades fitoplanctônicas e de alguns fatores abióticos em trecho do Rio Jacuí, Rio Grande do Sul, Brasil. Acta Botanica Brasilica 2(1-2) 31-46.

Scott, A.M.; Grönblad, R. \& Croasdale, H. 1965. Desmids from the Amazon Basin, Brazil. Acta Botanica Fennica 69: 1-93.

Soares, M.C.S.; Sophia, M.G. \& Huszar, V.L.M. 2007. Phytoplankton flora of two rivers in Southeast Brazil - Paraibuna and Pomba Rivers, Minas Gerais. Revista Brasileira de Botânica 30(3)433-450.

Sophia, M.G. 1991. Desmidias de hábito solitário (exceto Micrasterias C. Agardh ex Ralfs) do município do Rio de Janeiro e arredores, Brasil. Revista Brasileira de Biologia 51(1): 85-107.

Transeau, E.N. 1926. The genus Mougeotia. The Ohio Journal of Science 26: $311-338$.

Transeau, E.N. 1951. The Zygnemataceae: fresh algae conjugate algae. Columbus, The Ohio State University Press.

Ungaretti, I. 1981. Desmídias (Zygnemaphyceae) de um açúde do Morro Santana, Porto Alegre, Rio Grande do Sul, Brasil. Iheringia, Série Botânica 27: 3-26.

Wille, N. 1884. Bidrag til Syda merikas Algoflora, 1-3. Bihang till Kongliga Svenska Vetenskaps-akademiens handlingar 8:1-64.

Wittrock, V.B. \& Nordstedt, C.F.O. 1882. Algae aquae dulcis exsiccatae praecipue scandinavicae quas adjectis algis marinis chlorophyllaceis et phycochromaceis. Holmiae: O.L. Svanbäcks Boktryckeri Aktiebolac. Fasc. 10: exsic. no 451-500.

Zarina, A.; Masud-Ul-Hasan \& Mustafa, S. 2008. Taxonomic Studies of the class Zygnemophyceae Shameel from north-eastern areas of Pakistan. Pakistan Journal of Botany 40(6): 2561-2566. 